ROSIANE MATTAR ${ }^{1}$

ELIZA YOSHIKO KOCHI SIIVA ${ }^{2}$

LUIZ CAMANO ${ }^{3}$

ANelise RIEDEL ABRAHÃO ${ }^{4}$

Osmar Ribeiro Colás ${ }^{5}$

JORGE ANDALAFT NeTO ${ }^{6}$

UMBERTO GAZI LIPPI

\section{A violência doméstica como indicador de risco no rastreamento da depressão pós-parto}

\author{
Domestic violence as a risk factor in the screening of for \\ post-partum depression
}

Artigos originais

Palavras-chave

Depressão pós-parto Comportamento materno Transtornos de adaptação Período pós-parto Violência doméstica Indicador de risco

Keywords

Depression, postpartum Maternal behavior

Adjustment disorders Postpartum period Domestic violence Risk index

\title{
Resumo
}

OBJETIVO: apurar a freqüência de risco para depressão pós-parto (DP) em puérperas de hospital de São Paulo, região Sudeste do Brasil, e determinar fatores associados - entre eles a violência doméstica (VD). MÉTODOS: estudo descritivo, tipo corte transversal. Participaram 133 mulheres, que tiveram partos com idade gestacional de 20 semanas ou mais, no período de agosto a setembro de 2005, em maternidade terciária em São Paulo (Brasil). Foram entrevistadas empregandose a versão em português do Abuse Assessment Screen para o diagnóstico de violência e responderam questionário de auto-avaliação do risco de DP (Edinburgh Postnatal Depression Scale). As variáveis foram representadas por freqüências absoluta e relativa. A associação entre as variáveis de interesse e DP foi avaliada pelo teste do $\chi^{2}$ ou exato de Fisher. Adotou-se o nível de significância de 5\%. RESULTADOS: o risco de DP foi identificado em 24 puérperas (1 8\%). Das mulheres entrevistadas, 38,3\% referiram história de abusos. Observou-se associação entre a ocorrência de VD após os 15 anos de idade e o risco de depressão ( $\mathrm{p=0}, 03)$. A ocorrência de abusos no grupo de mulheres com probabilidade de apresentar DP foi de 58,3\%, proporção significativamente maior do que a observada no grupo controle com 33,9\%. CONCLUSÕES: a probabilidade de apresentar depressão foi alta entre as puérperas assistidas em maternidade terciária da região Sudeste do Brasil. A VD sofrida após os 15 anos de idade esteve estatisticamente associada ao risco de DP.

\section{Abstract}

PURPOSE: to assess the prevalence of the risk of post-partum depression in women in the post-natal ward of a hospital in São Paulo city, in the southeastern region of Brazil, and analyze the associated factors, including domestic violence (DV). METHODS: this was a descriptive, cross-sectional study. The participants were 133 women with at least 20 weeks of gestation age, who delivered their babies from August to September 2005 in a tertiary maternity in the city of São Paulo (Brazil). They were interviewed using the Portuguese version of the Abuse Assessment Screen for the diagnosis of violence and filled out a self-evaluation questionnaire for post-partum depression (Edinburgh Postnatal Depression Scale). Variables were presented as absolute and relative frequencies. The $\chi^{2}$ or Fisher exact tests were used to analyze possible associations between the variables of interest and post-partum depression. The value of $5 \%$ was considered significant. RESULTS: risk for post-partum depression was detected in 24 women (18\%). A total of 38.3\% of the participants interviewed had a history of abuse. There was an association between DV after they were 15 years old and risk of depression $(p=0.036)$. The prevalence of abuse in the group of women at risk for post-partum depression was $58.3 \%$ and this was significantly higher than the $33.9 \%$ observed in the control group. CONCLUSIONS: the probability of presenting depression was high among the post-partum women attended at a tertiary maternity in the southeast of Brazil. The DV after they were 15 years old was significantly associated with risk of post-partum depression.

Correspondência:

Rosiane Matta Rua Dr. Diogo de Faria, 477, apto. 31 - Vila Clementino CEP 04037-000 - São Paulo (SP) Fone: (11) 5571-1478 Fax: (11) 5579-3507 E-mail: rosiane.toco@epm.br

Recebido 26/03/2007

Aceito com modificações 20/09/2007
Setor de Puerpério da Enfermaria de Obstetrícia do Hospital do Servidor Público Estadual "Francisco Morato de Oliveira" - HSPE-FMO - São Paulo (SP), Brasil.

1 Livre-Docente, Professora Associada do Departamento de Obstetrícia da Escola Paulista de Medicina da Universidade Federal de São Paulo - UNIFESP - São Paulo (SP), Brasil.

2 Pós-graduanda do Departamento de Obstetrícia da Escola Paulista de Medicina da Universidade Federal de São Paulo - UNIFESP - São Paulo (SP), Brasil.

${ }_{3}^{3}$ Professor Titular do Departamento de Obstetrícia da Escola Paulista de Medicina da Universidade Federal de São Paulo - UNIFESP - São Paulo (SP), Brasil.

4 Professora Adjunta do Departamento de Enfermagem da Escola Paulista de Medicina da Universidade Federal de São Paulo UNIFESP - São Paulo (SP), Brasil.

${ }^{5}$ Médico Assistente do Departamento de Obstetrícia da Escola Paulista de Medicina da Universidade Federal de São Paulo - UNIFESP - São Paulo (SP), Brasil.

- Professor Titular da Universidade de Santo Amaro - UNISA - São Paulo (SP), Brasil; Professor Voluntário da Escola Paulista de Medicina da Universidade Federal de São Paulo - UNIFESP - São Paulo (SP), Brasil.

7 Diretor do Serviço de Ginecologia e Obstetrícia do Hospital do Servidor Público Estadual "Francisco Morato de Oliveira" - HSPE-

FMO - São Paulo (SP), Brasil; Professor Titular do Departamento de Obstetrícia da Universidade Metropolitana de Santos - UNIMES

- Santos (SP), Brasil. 


\section{Introdução}

O puerpério é fase especial na vida da mulher, durante a qual as atenções estão voltadas para o recémnascido (RN) e existe, por parte da família, expectativa de mudanças e alegrias.

Ressalte-se que, para a mãe, os cuidados e as necessidades da criança demandam tempo e trabalho, acompanhados, muitas vezes, de distúrbios do sono, cansaço e agitação. Essa sobrecarga física e as responsabilidades dos cuidados com o RN podem ocasionar ansiedade e, como conseqüência, estresse psicológico, que pode manifestar-se como um quadro de depressão ${ }^{1}$.

A depressão pós-parto (DP) foi definida como transtorno do humor que se inicia, normalmente, nas primeiras quatro semanas após o parto e pode ser de intensidade leve e transitória, neurótica, até desordem psicótica² ${ }^{2}$. A depressão maior ocorre por período mínimo de duas semanas, com a presença do humor depressivo ou anedonia (diminuição ou perda do interesse nas atividades anteriormente agradáveis) associada a pelo menos quatro dos sintomas: alteração significativa de peso ou do apetite, insônia ou sono excessivo, fadiga, agitação ou lentidão psicomotora, sentimentos de desvalia ou culpa, perda de concentração e idéias de morte ou suicídio².

A psicose puerperal tem incidência de um a dois casos em mil nascimentos ${ }^{3}$. Acarreta grave prejuízo da capacidade funcional da mulher e pode ocasionar o suicídio ou mesmo o infanticídio, geralmente associado a quadro de alucinação. A recorrência de psicose puerperal é de 30 a $50 \%$ a cada parto subseqüente ${ }^{3}$.

A DP tem prevalência de 10 a $15 \%$ em mulheres adultas ${ }^{3,4}$ e de até $26 \%$ entre as adolescentes ${ }^{2}$. Sua etiologia foi relacionada a fatores hormonais e hereditários 5 . $\mathrm{Na}$ gestação, os níveis de estrógeno e progesterona estão aumentados e podem ser responsáveis por alterações de humor observadas nesse período da vida da mulher. A redução brusca dos hormônios, que sucede o parto, estaria, também, envolvida na etiologia dos quadros depressivos ocorridos no puerpério ${ }^{3}$. Os fatores de risco descritos na literatura e associados à DP são: mães jovens, sem companheiro ou com pobre ajuste marital, baixo nível socioeconômico, baixa paridade, antecedente de depressão, personalidade negativa, depressão ou ansiedade durante a gravidez, experiência de eventos estressantes na gestação ou puerpério precoce ${ }^{6,7}$. Embora os transtornos mentais causem pouco mais de $1 \%$ de mortalidade, a depressão foi a doença que mais determinou a incapacitação em mulheres, tanto em países desenvolvidos como naqueles em desenvolvimento ${ }^{3}$. Nos episódios depressivos mais graves, a mulher pode apresentar agitação psicomotora intensa e idéia suicida ${ }^{3}$.
A DP pode ocasionar pensamentos obsessivos, envolvendo violência contra a criança ${ }^{3}$, e existe possibilidade de negligência nos cuidados infantis, inclusive diminuição do período de amamentação ${ }^{8}$. Incita sentimentos negativos, culpa e desinteresse pela criança e resultado insatisfatório da interação mãe-filho. Pode, portanto, causar impacto negativo no desenvolvimento do RN, tanto imediato como em longo prazo. Existem evidências de associação com distúrbios de comportamento nesses filhos aos três anos e defeito cognitivo aos quatro anos de idade ${ }^{6}$.

Muitos dos fatores relacionados ao desenvolvimento da DP já estão presentes durante a gravidez ${ }^{4}$. Estudos preliminares sugerem que o humor da puérpera no período pós-parto imediato pode predizer a ocorrência de depressão ${ }^{9}$, portanto pode-se detectar precocemente as mulheres com probabilidade de desenvolvê-la.

A violência doméstica (VD) tem sido relacionada à depressão e implicada, muitas vezes, como uma de suas causas, pois ela pode determinar repercussões sobre vários aspectos da vida da mulher: na área social, com isolamento progressivo e dificuldades no trabalho; na saúde, com a presença de vários sintomas como dores abdominais, cefaléias e lombalgias, ou agravo de enfermidades preexistentes, desordem pós-traumática, distúrbios psicossomáticos e alteração do sono; e associação com o uso de drogas lícitas ou ilícitas ${ }^{10,11}$.

Estima-se que um terço das mulheres agredidas sofrem depressão e que esta foi a responsável por cerca de um quarto das tentativas de suicídios entre as vitimizadas ${ }^{12}$. A violência contra a mulher foi definida como qualquer ato baseado no gênero que resulta, ou possa resultar, em danos físico, sexual, mental ou sofrimento, incluindo ameaça de praticar tais atos, coerção ou privação da liberdade que ocorre em espaço público ou na vida privada ${ }^{13}$.

Foi apurado que uma em cada cinco mulheres experimenta alguma forma de violência durante sua vida, podendo sofrer agressão grave e até a morte ${ }^{14}$. No Brasil, 2,1 milhões de mulheres sofreram espancamentos graves, com mais de 205 mil casos registrados nas Delegacias de Defesa da Mulher no ano de 2001, colocando o país no terceiro lugar entre os que mais agridem as mulheres ${ }^{15}$.

Durante a gestação, diversos tipos de associação com fenômenos violentos podem ser observados. Por um lado, a gravidez pode ser fator protetor contra a VD, observando-se a diminuição ou a cessação de episódios abusivos; por outro lado, ela pode determinar o início das agressões ou aumento de sua gravidade e freqüência, ou mesmo, não apresentar mudança no padrão abusivo ${ }^{16}$. Em cerca de $90 \%$ dos casos, os maus 
tratos já estavam presentes antes da gestação ${ }^{17,18}$, sendo que a história de VD pode ser o principal preditor de agressões na gravidez.

Ao estudarmos tal evento, observamos que algumas mulheres vitimizadas apresentavam alteração de humor no puerpério, o que nos levou a supor que a ocorrência de VD, quer seja na gestação ou anteriormente a ela, pode estar associada a maior risco de DP.

No puerpério, existem vários fatores que podem gerar estresse e ansiedade para a mulher, e o limite entre o que é normal ou fisiológico e o que é patológico pode ser difícil de avaliar ${ }^{5}$. Por outro lado, o diagnóstico e a determinação da gravidade da depressão são imprescindíveis para elaboração de diretrizes de tratamento e acompanhamento e, neste sentido, as ferramentas de avaliação podem ser úteis e práticas. Existem alguns instrumentos com essa finalidade, como a Edinburgh Postnatal Depression Scale (EPDS), questionário de auto-avaliação desenvolvido na Grã-Bretanha para pesquisa da DP, traduzido e validado em diversos países, inclusive no Brasil, em 1999, por Santos et al. ${ }^{19}$.

Eberhard-Gran et al. ${ }^{20}$ realizaram revisão dos estudos de validação da EPDS e verificaram que esta ferramenta mostrou elevada sensibilidade para a identificação da DP, na maioria das investigações. A EPDS, embora apresente algumas desvantagens, como a dificuldade para determinar a confiabilidade e a necessidade de o paciente ser cooperativo, sem psicopatologia grave e com grau mínimo de escolaridade ${ }^{21}$, tem sido o método mais usado, pois abarca vantagens como a econômica, necessita de pouco tempo para o seu preenchimento e tem boa aceitação por parte dos pacientes com transtornos de humor. Esse instrumento pode ser aplicado por profissionais não-especializados em saúde mental, como tocoginecologistas, enfermeiros e assistentes sociais, e pode predizer precocemente as mulheres com possibilidade de apresentar depressão, facilitando a intervenção terapêutica ${ }^{4,19}$.

Tendo em vista a importância do diagnóstico precoce da DP e a possível relação com a VD, tivemos como objetivos determinar a prevalência de mulheres com risco de DP, os fatores associados e se existe correlação com a VD ocorrida após os 15 anos de idade e na gravidez, em puérperas atendidas em um hospital localizado no município de São Paulo, na região Sudeste do Brasil, e que presta assistência ao funcionário público do Estado e seus dependentes.

\section{Métodos}

Estudo descritivo, tipo corte transversal, fundamentado nos dados obtidos de entrevistas de 133 mulheres internadas no período de agosto a setembro de 2005, o que correspondeu a $81 \%$ dos partos nesse período, no Setor de Puerpério da Enfermaria de Obstetrícia do Hospital do Servidor Público Estadual Francisco Morato de Oliveira (HSPE-FMO), que atende exclusivamente aos servidores públicos do Estado de São Paulo e seus dependentes.

Foram convidadas a participar da pesquisa todas as mulheres que deram à luz neste hospital durante o período de estudo. Utilizaram-se como critérios para inclusão: puérperas no segundo ou terceiro dia após o parto, com idade gestacional de 20 semanas completas ou mais ou RN com peso superior a $500 \mathrm{~g}$ e em condições de alta hospitalar; como critérios de exclusão: diagnóstico de gravidez múltipla, má formação fetal, puérperas que estivessem sob tratamento psicológico ou psiquiátrico e as que se recusassem a participar.

Para a caracterização sociodemográfica e coleta de antecedentes pessoais e obstétricos das puérperas, foram aplicados questionários estruturados e estudaram-se os prontuários para análise das ocorrências durante o pré-natal.

Avaliou-se a VD pelo instrumento Abuse Assessment Screen, que foi elaborado nos Estados Unidos, em 1989, para rastrear situações de violência sofridas pela mulher grávida, e que foi traduzido para o português por Reichenheim et al. ${ }^{22}$. Contém perguntas para identificar o tipo de abuso (físico, sexual e/ou emocional), a freqüência, a gravidade, os locais corporais das agressões físicas, bem como o agressor. Em nossa pesquisa, os eventos violentos foram agrupados e analisados, segundo sua ocorrência, em qualquer momento da vida da mulher após os 15 anos de idade ou durante a gravidez.

Em relação à depressão puerperal, utilizou-se questionário elaborado com base na EPDS, instrumento de auto-avaliação que contém dez perguntas com quatro opções que são pontuadas de 0 a 3 , de acordo com a presença ou intensidade dos sintomas: humor deprimido ou disfórico, distúrbio do sono, perda do prazer, diminuição do desempenho, culpa e idéias de morte e suicídio $^{19}$ (Quadro 1).

As entrevistadas foram consideradas como do grupo de risco para desenvolver depressão, se os escores alcançados na EPDS fossem iguais ou maiores que 10. Este ponto de corte foi fundamentado em estudo de validação realizado no Brasil ${ }^{19} \mathrm{com}$ mulheres entre a $6^{\mathrm{a}}$ e a $24^{\mathrm{a}}$ semanas pós-parto, por apresentar elevada sensibilidade (92\%), embora baixa especificidade $(66 \%)$, mas com eficiência global de $75 \%$ para a correta identificação de mulheres deprimidas.

Assim, determinamos dois grupos de mulheres: as que obtiveram escores $<10$ e as de risco de desenvolver 
DP com escores $\geq 10$ na EPDS. Analisamos também diferentes fatores que foram comparados entre esses dois grupos:

- demográficos: idade (adolescente ou não adolescente), cor/etnia, situação marital;

- socioeconômicos: escolaridade, possuir trabalho remunerado ou não, renda per capita;

- psicossociais: planejamento da gravidez atual ou não e se, após a confirmação da gravidez, esta foi desejada ou não;

- violência doméstica: abusos ocorridos após os 15 anos de idade e/ou na gravidez atual.

Foram pesquisados também os hábitos: tabagismo e alcoolismo. Foram definidos por meio do teste $\mathrm{Cage}^{23}$, que é um teste simples que identifica o alcoolismo por meio de quatro perguntas pelas quais se investiga a percepção que o indivíduo tem da necessidade de interromper ou diminuir sua ingestão alcoólica, o comportamento que a pessoa adota frente a críticas quanto à quantidade de álcool que ingere, a sensação de culpa pelo comportamento que apresenta quanto à ingestão de bebidas e a interferência da mesma sobre sua capacidade de trabalho. Quando dois destes aspectos se mostram presentes, firma-se o diagnóstico do alcoolismo.

Os antecedentes obstétricos investigados foram: paridade, ganho ponderal durante o pré-natal $<7$, de 7 a 20 e $\geq 20 \mathrm{~kg}$, trabalho de parto prematuro, descolamento prematuro de placenta, infecção do trato urinário, hipertensão arterial, diabetes mellitus, sangramento vaginal durante a gestação e idade gestacional ao parto.

Quadro 1 - Edinburgh Postnatal Depression Scale, para rastreamento de depressão pós-parto.

\begin{tabular}{|c|c|}
\hline \multicolumn{2}{|l|}{ 1. Eu tenho sido capaz de rir e achar graça das coisas } \\
\hline [ ] Como eu sempre fiz & [ ] Não tanto quanto antes \\
\hline [ ] Sem dúvida, menos que antes & [ ] De jeito nenhum \\
\hline \multicolumn{2}{|l|}{ 2. Eu sinto prazer quando penso no que está por acontecer em meu dia-a-dia } \\
\hline [ ] Como sempre senti & [ ] Talvez, menos que antes \\
\hline [ ] Com certeza menos & [ ] De jeito nenhum \\
\hline \multicolumn{2}{|l|}{ 3. Eu tenho me culpado sem necessidade quando as coisas saem erradas } \\
\hline [ ] Sim, na maioria das vezes & [ ] Sim, algumas vezes \\
\hline [ ] Não muitas vezes & [ ] Não, nenhuma vez \\
\hline \multicolumn{2}{|l|}{ 4. Eu tenho me sentido ansiosa ou preocupada sem uma boa razão } \\
\hline [ ] Não, de maneira alguma & [ ] Pouquíssimas vezes \\
\hline [ ] Sim, algumas vezes & [ ] Sim, muitas vezes \\
\hline \multicolumn{2}{|l|}{ 5. Eu tenho me sentido assustada ou em pânico sem um bom motivo } \\
\hline [ ] Sim, muitas vezes & [ ] Sim, algumas vezes \\
\hline [ ] Não muitas vezes & [ ] Não, nenhuma vez \\
\hline \multicolumn{2}{|c|}{ 6. Eu tenho me sentido esmagada pelas tarefas e acontecimentos do meu dia-a-dia } \\
\hline [ ] Sim. Na maioria das vezes eu não consigo lidar bem com eles & [ ] Sim. Algumas vezes não consigo lidar bem como antes \\
\hline [ ] Não. Na maioria das vezes consigo lidar bem com eles & [ ] Não. Eu consigo lidar com eles tão bem quanto antes \\
\hline \multicolumn{2}{|l|}{ 7. Eu tenho me sentido tão infeliz que eu tenho tido dificuldade de dormir } \\
\hline [ ] Sim, na maioria das vezes & [ ] Sim, algumas vezes \\
\hline [ ] Não muitas vezes & [ ] Não, nenhuma vez \\
\hline \multicolumn{2}{|l|}{ 8. Eu tenho me sentido triste ou arrasada } \\
\hline [ ] Sim, na maioria das vezes & [ ] Sim, muitas vezes \\
\hline [ ] Não muitas vezes & [ ] Não, de jeito nenhum \\
\hline \multicolumn{2}{|l|}{ 9. Eu tenho me sentido tão infeliz que eu tenho chorado } \\
\hline [ ] Sim, quase todo o tempo & [ ] Sim, muitas vezes \\
\hline [ ] De vez em quando & [ ] Não, nenhuma vez \\
\hline \multicolumn{2}{|l|}{ 10. A idéia de fazer mal a mim mesma passou por minha cabeça } \\
\hline [ ] Sim, muitas vezes, ultimamente & [ ] Algumas vezes nos últimos dias \\
\hline [ ] Pouquíssimas vezes, ultimamente & [ ] Nenhuma vez \\
\hline
\end{tabular}

Nota: nas questões 1, 2, e 4 a pontuaç̃ão para a sequiência é 0, 1, 2, 3. Nas demais, a pontuação é 3, 2, 1, 0 . 
Os fatores relacionados ao pai do $\mathrm{RN}$ também foram analisados e empregados para comparação entre os dois grupos. Assim, incluímos as variáveis do pai do RN, como idade (adolescente ou não adolescente), diferença de idade do casal ( $<10$ e $\geq 10$ anos), anos de estudo, possuir trabalho remunerado ou não, uso de tabaco e freqüência de embriaguez (nunca, menos de uma vez por semana, uma vez por semana e mais de uma vez por semana).

As informações foram colocadas em planilha do Microsoft Excel e analisadas pelo Software Estatístico SPSS para Windows, versão 10.0, para construção de tabelas e gráficos.

$\mathrm{Na}$ análise estatística, as variáveis qualitativas foram representadas por freqüências absoluta e relativa e as quantitativas por média, desvio padrão, mediana e valores mínimo e máximo. A presença de associação entre as variáveis qualitativas foi pesquisada pelo teste do $\chi^{2}$ ou exato de Fisher. Para cada variável estudada, calculou-se o risco relativo seguido do intervalo com $95 \%$ de confiança. O nível de significância adotado foi de $0,05(\alpha=5 \%)$.

Todas as participantes incluídas nesta pesquisa foram informadas sobre o estudo e seu caráter sigiloso; concordaram em participar e assinaram o termo de consentimento. Este estudo teve aprovação do Comitê de Ética em Pesquisa da Universidade Federal de São Paulo (Unifesp) e do HSPE-FMO.

Quando o abuso foi revelado durante a entrevista ou considerou-se a probabilidade de DP ou suicídio, foi proposto o encaminhamento da paciente para o Grupo de Apoio e Prevenção de Violência Doméstica do HSPE-FMO (GAPREV).

\section{Resultados}

Participaram 133 puérperas com média de idade de 28,8 anos $( \pm 7,7)$ e faixa de variação entre 15 e 46 ano). Eram adolescentes 16,5\% da amostra. Todas eram alfabetizadas e quase a totalidade $(92,5 \%)$ tinha, pelo menos, o primeiro grau completo. A maioria (82\%) convivia com parceiro em união legal ou consensual está-

Tabela 1 - Distribuição das puérperas, conforme pontos obtidos na escala de Edinburgh para avaliação do risco de depressão pós-parto.

\begin{tabular}{lcc}
\hline Pontos na escala de Edinburgh & N & $\%$ \\
\hline$<10$ (Grupo controle) & 109 & 82,0 \\
$\geq 10$ & 24 & 18,0 \\
$\geq 11$ & 17 & 12,8 \\
$\geq 12$ & 14 & 10,5 \\
$\geq 13$ & 11 & 8,3 \\
$\geq 14$ & 9 & 6,8 \\
\hline
\end{tabular}

vel, 70,7\% possuíam trabalho remunerado e $63,1 \%$ com renda per capita de pelo menos um salário-mínimo.

Dentre as mulheres entrevistadas, $51(38,3 \%)$ haviam sofrido abuso doméstico em algum momento da vida após os 15 anos de idade. A maioria (94\%) delas referiu a violência emocional como a mais freqüentemente sofrida e que foi perpetrada pelo companheiro em $56 \%$ dos casos, $10 \%$ pelo pai e $6 \%$ pela mãe. Dez mulheres $(7,5 \%)$ relataram abuso físico antes da gravidez, principalmente pelo companheiro (sete casos), sendo que os abusos relatados variaram desde tapas, palmadas, espancamento até esganadura.

Durante a atual gestação, cinco puérperas $(3,7 \%)$ foram agredidas fisicamente (tapas, manchas roxas, socos, até ferimento por arma branca). Todas elas já sofriam abusos antes da gestação. Três puérperas relataram repetidos estupros (três vezes ou mais) durante a gravidez pelo atual companheiro.

Em relação à pontuação da EPDS, a mediana foi 5 . Vinte e quatro puérperas tiveram escores $\geq 10$ da EPDS, o que representou $18 \%$ da amostra com probabilidade de vir a desenvolver DP (Tabela 1).

Comparando-se pacientes com escores $<10$ ou $\geq 10$ e as variáveis maternas (idade, cor/etnia, situação marital, escolaridade, possuir trabalho remunerado e renda per capita), hábitos (tabagismo, alcoolismo), história reprodutiva (paridade, gravidez planejada ou não), ocorrências observadas durante a gravidez (ganho ponderal, trabalho de parto prematuro, descolamento prematuro de placenta, infecção do trato urinário, hipertensão arterial, diabetes mellitus, sangramento na gravidez) e idade gestacional do parto, não notamos diferenças significantes ( $p>0,05$ para todas as comparações) entre os grupos (Tabela 2). Também não observamos associação entre os dois grupos quanto ao planejamento da gravidez ou não e se ela era desejada ou não.

Entre as mulheres com escore $\geq 10$ na EPDS, a referência de VD em algum momento da vida após os 15 anos foi de 58,3\% e, durante a gravidez, 8,3\%. Observamos associação significante entre a VD ocorrida após os 15 anos de idade e o de risco de depressão $(\mathrm{p}=0,03)($ Tabela 3).

Os eventos abusivos durante a gravidez não se mostraram significativamente relacionados à depressão, embora entre as mulheres com possibilidade de DP, $8,3 \%$ haviam sofrido agressões na gravidez contra $2,8 \%$ entre as puérperas do grupo controle.

Os fatores relacionados ao pai do RN (idade, diferença de idade do casal, tempo de escolaridade, exercício remunerado, uso de tabaco e álcool) foram analisados e não se notaram associações significativas com o risco de DP. 
Tabela 2 - Variáveis maternas, de acordo com a pontuação na escala de Edinburgh para avaliação do risco de depressão pós-parto.

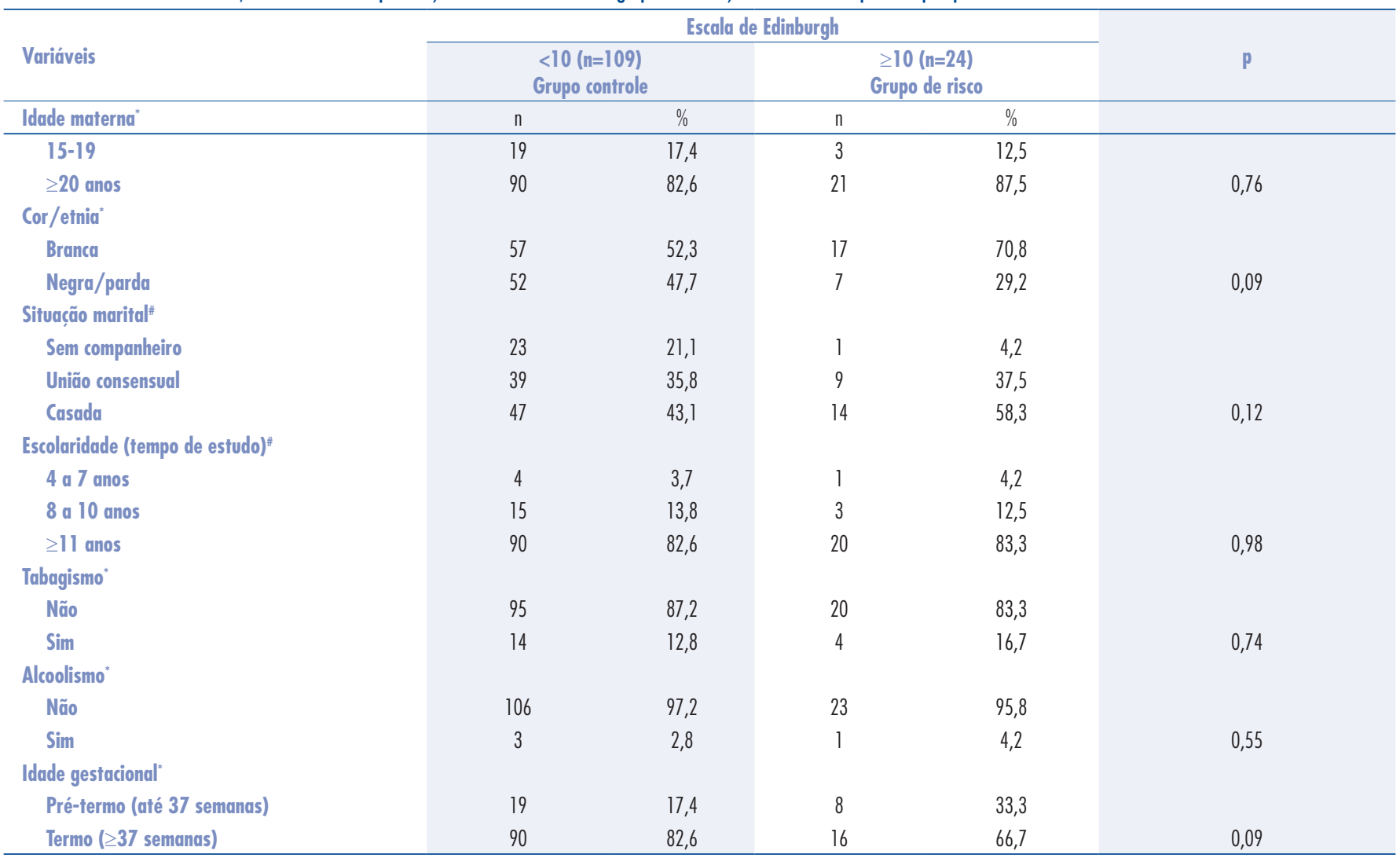

"Teste exato de Fisher; " teste do $\chi^{2}$.

Tabela 3 - Violência doméstica ocorrida após os 15 anos de idade e na gravidez, de acordo com pontuação na escala de Edinburgh para avaliação do risco de depressão pós-parto.

\begin{tabular}{|c|c|c|c|c|c|}
\hline \multirow{3}{*}{$\begin{array}{l}\text { Violência doméstica } \\
\text { Após os } 15 \text { anos de idade }\end{array}$} & \multicolumn{4}{|c|}{ Escala de Edinburgh } & \multirow{3}{*}{$p$} \\
\hline & \multicolumn{2}{|c|}{$\begin{array}{c}<10 \\
\text { Grupo controle (n=109) }\end{array}$} & \multicolumn{2}{|c|}{$\begin{array}{c}\geq 10 \\
\text { Grupo de risco (n=24) }\end{array}$} & \\
\hline & $n$ & $\%$ & $n$ & $\%$ & \\
\hline Não & 72 & 66,1 & 10 & 41,7 & \\
\hline Sim & 37 & 33,9 & 14 & 58,3 & $0,03^{*}$ \\
\hline \multicolumn{6}{|l|}{ Durante a gravidez } \\
\hline Não & 106 & 97,2 & 22 & 91,7 & \\
\hline Sim & 3 & 2,8 & 2 & 8,3 & 0,22 \\
\hline
\end{tabular}

"Teste do $\chi^{2}$.

\section{Discussão}

A freqüência apurada de risco de depressão no segundo ou terceiro dia após o parto foi $18 \%$, utilizando-se como critério de diagnóstico o valor da EPDS $\geq 10$.

No Japão ${ }^{24}$, em estudo realizado com o uso do mesmo instrumento no quinto dia do puerpério, observou-se que $20 \%$ das mulheres com escores 9 ou mais, nesta fase, apresentaram significativa correlação com quadro clínico de depressão um mês após o parto.

A porcentagem de mulheres sob risco em nosso estudo foi menor que o observado em investigação realizada na França ${ }^{25}$, que aplicou a EPDS no segundo ou terceiro dia após o parto, com reavaliação da quarta à sexta semana. Observou-se que o melhor ponto de corte para detectar precocemente mulheres com possibilidade de depressão, naquela população, foi o de escores de 10 a 11 (25,6\% das puérperas). Sugeriram sua aplicação rotineiramente na maternidade por apresentar boa validade, aplicação rápida e barata.

Pesquisa conduzida no Brasil ${ }^{19}$ considerou o ponto de corte 11/12 como o de maior adequação para o diagnóstico de DP no contexto do nosso país. Utilizando escores de 11/12 da EPDS, as prevalências de DP mencionadas foram de $12 \%$ no terceiro mês após o parto ${ }^{2}, 37,1 \%$ entre 12 e 16 semanas do puerpério ${ }^{6}$ e de $19,1 \%$ com 30 a 45 dias após o nascimento, em investigação de base populacional ${ }^{26}$.

A elevada freqüência de DP observada por nós e por outras pesquisas nos levaria a esperar que grande número de puérperas, ao retornar às consultas de avaliação pós-parto ou de planejamento familiar, descrevesse sintomas de depressão e tivesse o diagnóstico da patologia firmado. Este fato, entretanto, não é observado na prática clínica, talvez porque as pacientes se sintam envergonhadas e não relatem seus sentimentos e sintomas. 
Acreditamos que a DP deve ser tratada como problema de saúde pública e que cabe ao médico tocoginecologista a função de investigar ativamente quadros de depressão; no entanto, esses profissionais precisam ser sensibilizados e capacitados para assumir esta importante responsabilidade, que tem repercussão, não somente do ponto de vista da saúde da mulher, mas também social e econômica. Além disso, fundamentados na elevada prevalência dessa ocorrência, julgamos que há necessidade de que as autoridades da área da saúde promovam ações educativas-preventivas primárias.

Consideramos também que a grande vantagem da aplicação do questionário logo após o parto, quando a paciente ainda se encontra na maternidade, é a possibilidade de identificar precocemente mulheres com risco de desenvolverem DP ou que já se mostrem deprimidas. Embora complicações maternas e fatores clínicos possam modificar o humor no período pós-natal recente, há evidências que sugerem que essa alteração, já nesta fase, possa ser preditiva de $\mathrm{DP}^{9,24,25}$, permitindo a intervenção secundária rápida e minimizando as seqüelas.

Outro fator que demonstra a importância da busca ativa da DP é que vários outros critérios não se mostraram relevantes para detecção do risco, como: idade, cor/etnia, escolaridade, situação marital, paridade, trabalho remunerado e renda per capita. Este resultado é semelhante ao de outro estudo realizado na cidade de São Paulo com 70 puérperas entre a $12^{\mathrm{a}}$ e a $16^{\mathrm{a}}$ semanas após o parto, atendidas pelo Programa de Saúde da Família e que alcançaram escores 11/12 da EPDS ${ }^{2}$. Estes dados sugerem que não existem indicadores sociodemográficos que caracterizem as puérperas que apresentarão depressão, ou seja, estes quadros podem acometer a mulher independente da idade, etnia ou classe social a que pertence.

Em estudo ${ }^{25}$ realizado na França, os parâmetros idade, paridade, nível educacional e classe social também não se mostraram diferentes entre as mulheres com risco de depressão e as não-deprimidas.

Em nossa pesquisa, os hábitos maternos antes ou durante a gestação (tabagismo e alcoolismo), gravidez planejada ou não, desejada ou indesejada, ganho de peso e intercorrências na gestação (trabalho de parto prematuro, descolamento de placenta, infecção do trato urinário, parto prematuro) foram averiguados e não apuramos associações significantes.

Observamos, porém, relevante associação de história de VD na vida da mulher e o risco de DP. Em trabalho realizado na China ${ }^{27}$, utilizando os mesmos instrumentos de avaliação de VD e de DP, esta relação também foi observada e os autores concluíram que o efeito da agressão nas gestantes chinesas determina, principalmente, danos psicológicos. Estudo-piloto norte-americano relatou que mulheres com história recente ou passada de abuso físico ou sexual apresentaram maiores taxas de DP na primeira semana, no segundo e no terceiro mês após o parto do que as não agredidas ${ }^{28}$.

A agressão pode ocasionar na mulher sentimentos como vergonha, medo, culpa, baixa auto-estima e, conseqüentemente, o isolamento social, a ansiedade e a depressão. Geralmente, as vítimas de VD se isolam e não recebem suporte familiar, o que já foi aceito como fator de proteção contra quadros de $\mathrm{DP}^{7}$.

Nossa pesquisa foi realizada com mulheres de nível socioeducacional diferenciado em relação ao restante do país, e o questionário foi aplicado durante internação na enfermaria após o parto, onde os cuidados com o $\mathrm{RN}$ foram partilhados com a equipe de enfermagem. Trata-se de local comumente alegre, com ambiente positivo e descontraído, o que poderia influenciar as respostas e subestimar os dados obtidos. Mesmo assim, o resultado alcançado coloca a DP como uma das morbidades mais prevalentes dentro do ciclo gravídico-puerperal. Outras considerações sobre este trabalho merecem destaque, como as limitações metodológicas inerentes a todo estudo de prevalência que não permite inferir causalidade e o fato de que não podemos generalizar nossos achados, pois devemos considerar a casuística modesta e a população com base hospitalar e exclusivamente formada por servidores públicos e dependentes.

Julgamos que a investigação realizada na enfermaria, por outro lado, permitiu observar a puérpera e a interação mãe-filho. A aplicação da EPDS mostrou-se fácil, possibilitou o diagnóstico precoce de pacientes com probabilidade de apresentar depressão e, assim, o planejamento de estratégias de intervenção. A aplicação deste questionário (EPDS) deveria, portanto, ser incorporada à rotina nas enfermarias de puerpério, com o objetivo de promover ações para diminuir as conseqüências deletérias dessa depressão nas relações materno-infantis, familiares e sociais.

\section{Agradecimentos}

À psiquiatra Dra. Janete Simiema, às psicólogas Aparecida Bastos Pereira, Eva Wongtschowski e Marina Cardoso Smith Eberlein, às assistentes sociais Ana Maria de Souza, Rosemary Silva e Virginia Maria Quaresma, integrantes do GAPREV. À Dra. Maria Regina Torloni. 
1. Andreoli SB, Blay SL, Mari JJ. Escalas de rastreamento aplicadas na população geral. In: Gorestein C, Andrade LHSG, Zuardi AW, editores. Escalas de avaliação clínica em psiquiatria e psicofarmacologia. São Paulo: Lemos; 2000. p. 53-8.

2. Cruz EBS, Simões GL, Faisal-CuryA. Rastreamento da depressão pós-parto em mulheres atendidas pelo Programa de Saúde da Família. Rev Bras Ginecol Obstet. 2005;27(4):181-8.

3. Andrade LHSG, Viana MC, Silveira CM. Epidemiologia dos transtornos psiquiátricos na mulher. Rev Psiquiatr Clín (São Paulo). 2006;33(2):43-54.

4. Mallikarjun PK, Oyebode F. Prevention of postnatal depression. J R Soc Health. 2005; 125(5):221-6.

5. Camacho RS, Cantinelli FS, Ribeiro CS, Cantilino AY, Gonsales $B K$, Braguittoni $E$, et al. Transtornos psiquiátricos na gestação e no puerpério: classificação, diagnóstico e tratamento. Rev Psiquiatr Clín (São Paulo). 2006;33(2):92-102.

6. Da-Silva VA, Moraes-Santos AR, Carvalho MS, Martins MLP, Teixeira NA. Prenatal and postnatal depression among low income Brazilian women. Braz J Med Biol Res. 1998;31 (6):799-804.

7. Xu FS, Liu JX, Zhang SP, Li J, Su Q. Effects of intervening measures on postpartum depression. Zhonghua Fu Chan Ke Za Zhi. 2003;38(12):724-6.

8. Medeiros PPV, Furtado EF. Perfil dos cuidados maternos em mães deprimidas e não-deprimidas no período puerperal. J Bras Psiquiatr. 2004;53(4):227-34.

9. Dennis $\mathrm{CL}$. Can we identify mothers at risk for postpartum depression in the immediate postpartum period using the Edinburgh Postnatal Depression Scale? J Affect Disord. 2004;78(2):163-9.

10. Amaro H, Fried LE, Cabral H, Zuckerman B. Violence during pregnancy and substance use. Am J Public Health. 1990;80(5):575-9.

11. Berenson $A B$, Wiemann $C M$, Wilkinson GS, Jones WA, Anderson $G D$. Perinatal morbidity associated with violence experienced by pregnant women. Am J Obstet Gynecol. 1994; 170(6):1760-9.

12. Guth AA, Pachter L. Domestic violence and the trauma surgeon. Am J Surg. 2000; 179(2): 134-40.

13. Menezes TC, Amorim MMR, Santos LC, Faúndes A. Violência física doméstica e gestação: resultados de um inquérito no puerpério. Rev Bras Ginecol Obstet. 2003;25(5):309-16.

14. World Health Organization (WHO) Addressing violence against women and achieving the Millennium Development Goals. WHO [text on the Internet]. 2005 [cited 2006 Apr 12]. Available from: www.who.int/gender/documents/MDGs\&VAWSept05.pdf
15. Turazi D. Brasil vive "guerra doméstica": quatro mulheres são espancadas por minuto no país. Agência Brasil [texto na Internet]. 2004 [citado 2004 Maio 1 1]. Disponível em: http://www.radiobras. gov.br/centro\%20imprensa/mulher/mat_mulher6.php

16. Hedin LW. Postpartum, also a risk period for domestic violence. Eur J Obstet Gynecol Reprod Biol. 2000;89(1):41-5.

17. McFarlane J. Battering during pregnancy: tip of an iceberg revealed. Women Health. 1989; 15(3):69-84.

18. Stewart DE, Cecutti A. Physical abuse in pregnancy. CMAJ. 1993; 149(9): 1257-63.

19. Santos MFS, Martins FC, Pasquali L. Escalas de auto-avaliação de depressão pós-parto: estudo no Brasil. Rev Psiquiatr Clín (São Paulo). 1999;26(2):90-5.

20. Eberhard-Gran M, Eskild A, Tambs K, Opjordsmoen S, Samuelsen SO. Review of validation studies of the Edinburgh Postnatal Depression Scale. Acta Psychiatr Scand. 2001;104(4):243-9.

21. Calil MH, Pires MLN. Aspectos gerais das escalas de avaliação de depressão. In: Gorestein C, Andrade LHSG, Zuardi AW, editores. Escalas de avaliação clínica em psiquiatria e psicofarmacologia. São Paulo: Lemos; 2000. p. 65-9.

22. Reichenheim ME, Moraes CL, Hasselmann MH. Equivalência semântica da versão em português do instrumento Abuse Assessment Screen para rastrear a violência contra a mulher grávida. Rev Saúde Pública. 2000;34(6):610-6.

23. Pereira MG. Epidemiologia: teoria e prática. Rio de Janeiro: Guanabara Koogan; 2001 . p. 358-76.

24. Yamashita H, Yoshida K. Screening and intervention for depressive mothers of new-born infants. Seishin Shinkeigaku Zasshi. 2003; 105(9): 11 29-35.

25. Teissedre F, Chabrol H. Detecting women at risk for postnatal depression using the Edinburgh Postnatal Depression Scale at 2 to 3 days postpartum. Can J Psychiatry. 2004; 49(1):51-4.

26. Moraes IGS, Pinheiro RT, Silva RA, Horta BL, Sousa PLR, Faria $A D$. Prevalência da depressão pós-parto e fatores associados. Rev Saúde Pública. 2006;40(1):65-70.

27. Leung WC, Kung F, Lam J, Leung TW, Ho PC. Domestic violence and postnatal depression in a Chinese community. Int J Gynaecol Obstet. 2002;79(2):159-66.

28. Records K, Rice M. A comparative study of postpartum depression in abused and non-abused women. Arch Psychiatr Nurs. 2005; 19(6):281-90. 